Article

\title{
Development and Evaluation of Light-Weight Active Noise Cancellation Earphones
}

\author{
Sen M. Kuo ${ }^{1}$, Yi-Rou Chen ${ }^{1}$, Cheng-Yuan Chang ${ }^{1, *}$ and Chien-Wen Lai ${ }^{2}$ \\ 1 Department of Electrical Engineering, Chung Yuan Christian University, Taoyuan 320, Taiwan; \\ kuo1065@gmail.com (S.M.K.); christineub1103201506@gmail.com (Y.-R.C.) \\ 2 Changhua Christian Hospital, Changhua 500, Taiwan; 70672@cch.org.tw \\ * Correspondence: ccy@cycu.edu.tw; Tel.: +886-3-265-4838
}

Received: 26 May 2018; Accepted: 17 July 2018; Published: 19 July 2018

\begin{abstract}
This paper presents the development of active noise control (ANC) for light-weight earphones, and proposes using music or natural sound to estimate the critical secondary path model instead of extra random noise. Three types of light-weight ANC earphones including in-ear, earbud, and clip phones are developed. Real-time experiments are conducted to evaluate their performance using the built-in microphone inside KEMAR's ear and to compare with commercially-available ANC headphones and earphones. Experimental results show that the developed light-weight ANC earphones achieve higher noise reduction than the commercial ANC headphones and earphones, and the in-ear ANC earphone has the best noise reduction performance.
\end{abstract}

Keywords: active noise control; light-weight earphone; natural sound; secondary path model

\section{Introduction}

Portable devices like smart phones and MP3 players with wearable headphones or earphones are widely used by every age group from youth to the elderly. These portable devices bring convenience to people for communication and entertainment in any environment, for example in public buildings and on transportation [1]. Unfortunately, the performance of these devices will be degraded by annoying environmental noise. The acoustic noise problem has become more serious as increased numbers of equipment such as engines, blowers, fans, air-conditioners, and compressors are used in many installations, air planes, automobiles, etc. [2].

In general, there are two ways to reduce the environmental noise for portable devices: passive and active. The passive method uses thick acoustic earmuffs to cover the ear completely to block the environmental noise. However, they are relatively large, bulky, costly, and ineffective at low frequency range. The active method uses the ANC system to cancel the unwanted noise based on the principle of superposition [3]. Specifically, an anti-noise of equal amplitude and opposite phase is generated and actively combined with the primary noise, thus resulting in the cancellation of both noises.

Several studies were published on the application of ANC headphones [4-11]. In addition, several ANC headphones are commercially available and have been carefully evaluated [12]. Basically, there are three types of ANC headphones on the market. The first type of headphone (type A) has earmuffs that sit on top of the ears. The second type (type B) completely encloses the ears. These two types of ANC headphones are over-ear headphones and rely on large and bulky passive earmuffs clamping on the ears to reduce high frequency noise, thus they are not portable while performing outdoor activities. Furthermore, these passive headphones are not comfortable to wear due to the pressure experienced on the side of the head restricting blood flow and hence producing discomfort over time [13]. Users experience mild headaches, feel headphones pushing the ears against the head (benign paroxysmal positional vertigo [14]), and feel clamping pressure 
on their eardrum. The last type is an in-ear ANC earphone (type C) which was developed recently. Therefore, light-weight ANC earphones are the future trend; they are more comfortable and they are efficient for users to reduce environmental noise.

As shown in Figure 1, this paper develops three different light-weight ANC earphones: in-ear, earbud, and clip phones, and compares their noise reduction with commercially available ANC headphones (types A, B and C) of a leading brand company. The rest of the paper is organized as follows. Section II introduces the ANC algorithms used for the earphones and proposes using natural sound for modeling the required secondary path. Section III presents real-time experimental results and compares and analyzes their performance.

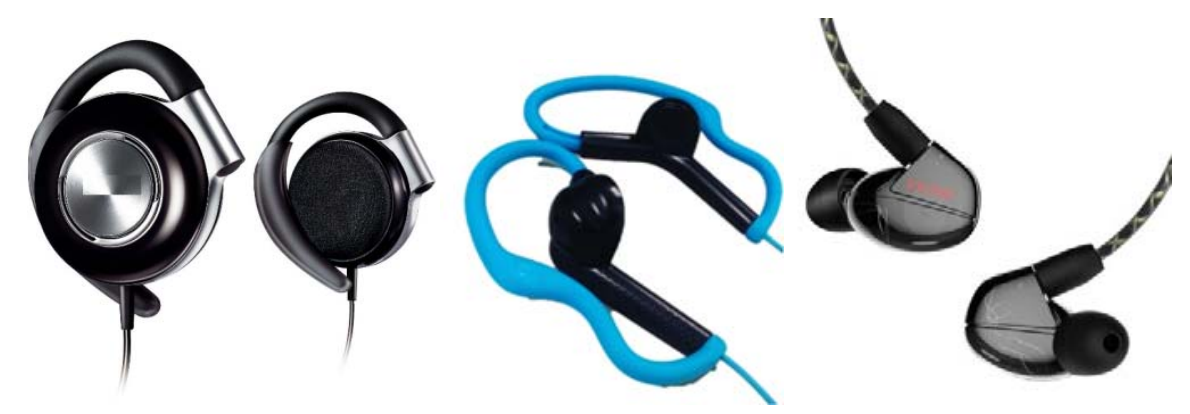

Figure 1. Three types of earphones: clip earphone (left), earbud earphone (middle), and in-ear earphone (right).

\section{ANC Algorithms for Earphones}

This section introduces the single-channel adaptive feedback ANC algorithm, which requires only one error sensor for each side of the earphone.

\subsection{Adaptive Feedback ANC Algorithm}

The basic principle of the adaptive feedback ANC is to estimate the primary noise to be canceled to use it as the reference signal $x(n)$ for the adaptive filter $W(z)$. As shown in Figure 2, the secondary signal $y(n)$ generated by the adaptive filter is filtered by the secondary path model $\hat{S}(z)$ and then added with $e(n)$ measured from the error sensor to synthesize the reference signal $x(n)$. The secondary signal $y(n)$ is generated as

$$
y(n)=\sum_{l=0}^{L-1} w_{l}(n) x(n-l)
$$

where $w_{l}(n),(l=0,1, \cdots, L-1)$ are the coefficients of $W(z)$ at time $n$, and $L$ is the filter length. These filter coefficients are updated by the filtered-X least-mean-square (FXLMS) algorithm [3] expressed as

$$
w_{l}(n+1)=w_{l}(n)+\mu x^{\prime}(n-l) e(n)
$$

where $\mu$ is the step size. The filtered signal is

$$
x^{\prime}(n) \equiv \sum_{m=0}^{M-1} \hat{s}_{m}(n) x(n-m)
$$

where $\hat{s}_{m}(n), \quad(m=0,1, \cdots, M-1)$ are the coefficients of the FIR filter $\hat{S}(z)$ (with length $\left.M\right)$ that is the secondary path estimate. Figure 2 clearly shows that the reference signal $x(n)$ equals the primary noise $d(n)$ if the perfect secondary model is available, i.e., $\hat{S}(z) \cong S(z)$. The method of modeling the secondary path is presented in the following subsection. 


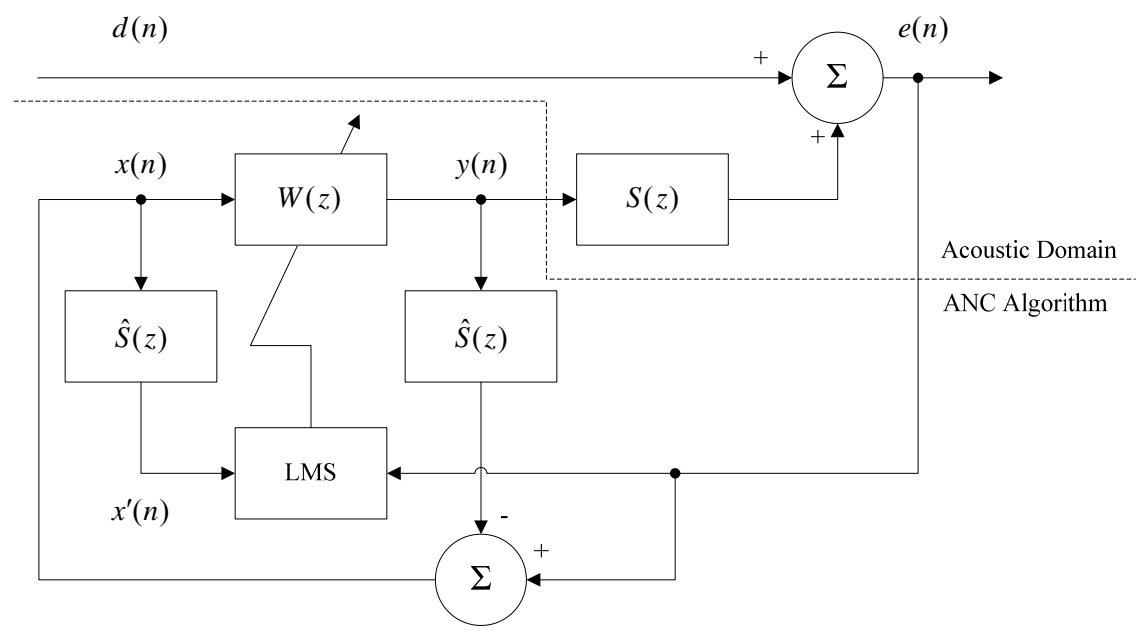

Figure 2. Feedback ANC algorithm.

\subsection{Natural Sound for Secondary Path Modeling}

As shown in Figure 2, the adaptive feedback ANC algorithm requires the secondary path model $\hat{S}(z)$ for synthesizing the reference signal $x(n)$, and compensating the effects of secondary path $S(z)$ for updating $W(z)$. The most popular method for estimating the secondary path is adaptive system identification using a white noise as the excitation signal. Unfortunately, extra random noise is undesired for consumer electronics such as earphones. Therefore, this paper proposes to use natural sound instead of white noise for the secondary path modeling.

Figure 3 shows the block diagram of using audio signal for the secondary path modeling. The natural sound signal $a(n)$ stored in memory is used as the excitation signal to drive the loudspeaker, and it also served as the input signal for the adaptive filter $\hat{S}(z)$. The desired signal $b(n)$ is received by the error sensor.

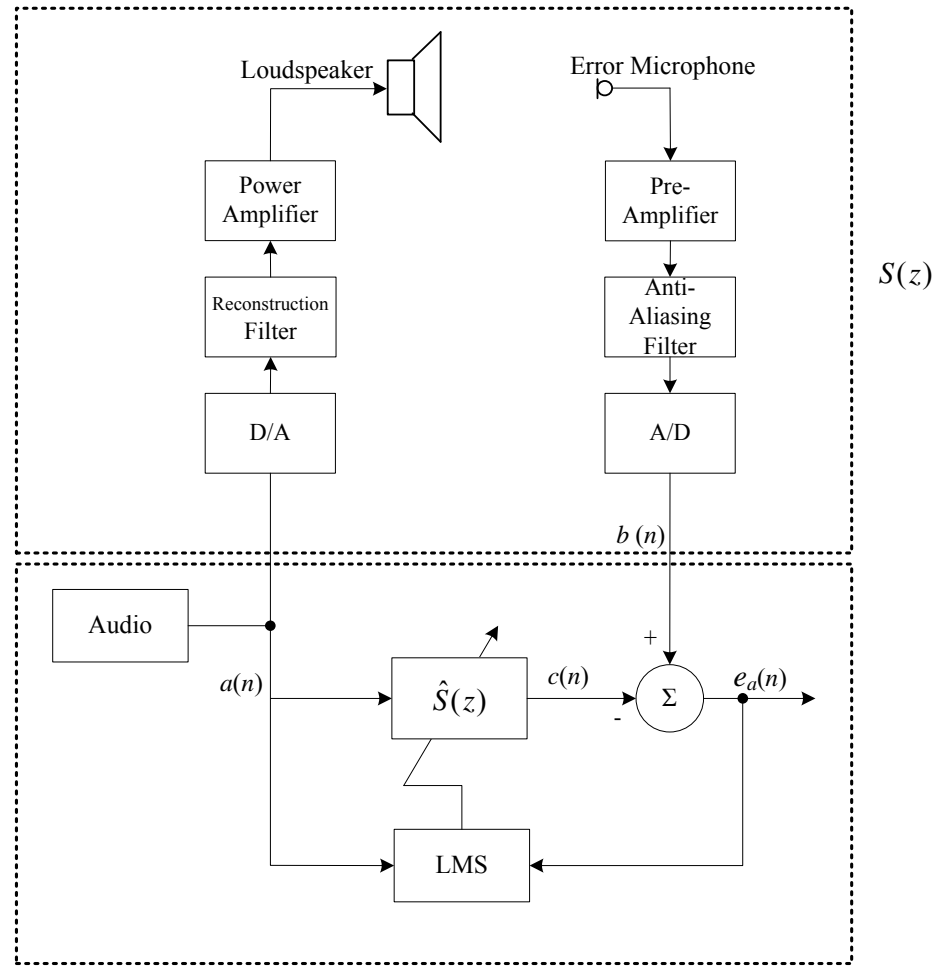

Figure 3. Block diagram of using natural sound for secondary path modeling. 
The modeling error signal is computed as

$$
e_{a}(n)=b(n)-c(n)
$$

where $c(n)$ is computed as

$$
c(n)=\sum_{m=0}^{M-1} \hat{s}_{m}(n) a(n-m)
$$

In (5), $\hat{s}_{m}(n)$ is the $m$-th coefficient of the secondary path estimation filter $\hat{S}(z)$ at time $n$. The adaptive filter is updated using the traditional least mean square (LMS) algorithm,

$$
\hat{s}_{m}(n+1)=\hat{s}_{m}(n)+\mu a(n-m) e_{a}(n), m=0,1, \cdots, M-1
$$

Both the reconstruction and anti-aliasing filters are lowpass filters.

It is important to note that Figures 2 and 3 can be combined to form the audio integrated ANC algorithm for on-line modeling of secondary path using the audio signal played by the ANC earphones [15].

\section{Real-Time Experiments}

This section presents real-time experimental results showing noise reduction achieved by the ANC earphones and compares them with commercial-available ANC earphones and headphones.

\subsection{Experimental Setups}

At first, we attached an error microphone each to our in-ear, earbud and clip earphones. Figure 4a shows the example of the clip earphone with the attached microphone. For the testing setup shown in Figure $4 b$, a loudspeaker is used as a noise source to generate the primary noise. The speaker is located $20 \mathrm{~cm}$ away from the KEMAR's right ear. The loudspeaker inside the light-weight earphone is used as the secondary loudspeaker to generate the anti-noise, and a MEMS microphone serving as the error microphone is attached on a suitable location of the earphone that is close to the ear canal. A digital signal processing (DSP) development platform (Texas Instrument, TMS320C6713 DSK) is used for implementing the adaptive feedback ANC algorithm. The ADC and DAC card (Heg Co. Ltd., 6713IFB, Mandideep, India) is used to do the signal conversion. The sampling rate is $8 \mathrm{kHz}$, and the cutoff frequency of both the lowpass filters (the reconstruction and anti-aliasing filters) is $3.2 \mathrm{kHz}$. The experimental results are measured by the built-in microphone inside the KEMAR's ear, which has input impedance closely resembling that of an average human ear.

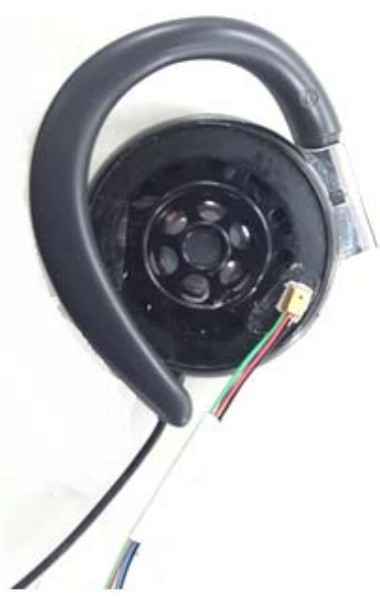

(a)

Figure 4. Cont. 


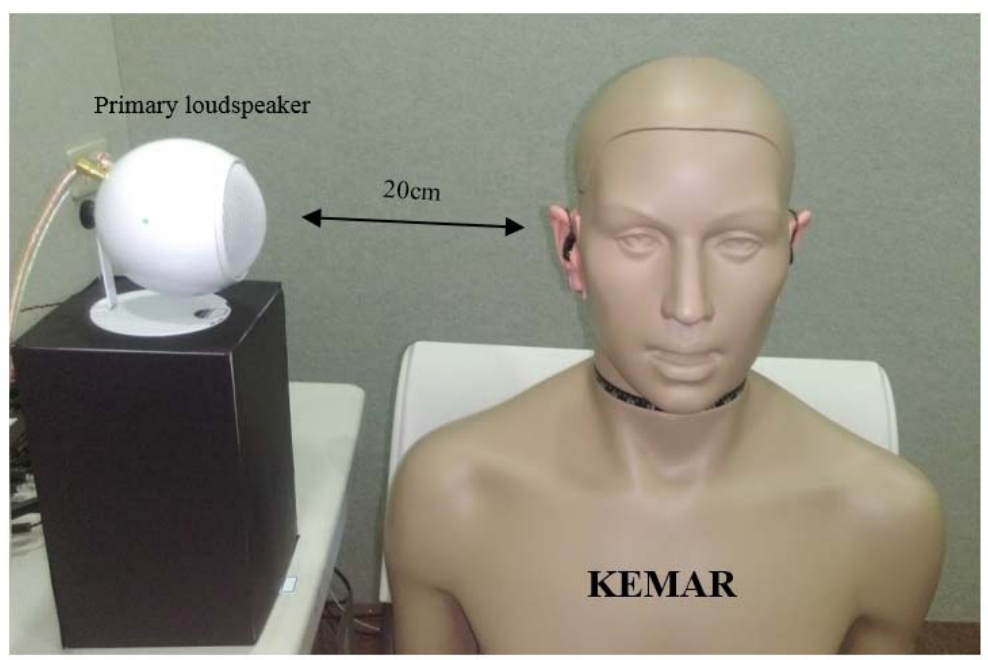

(b)

Figure 4. Experimental setup (a) for the clip earphone with attached error microphone (b) for testing ANC earphones and headphones.

The pinna of KEMAR's ear is a soft rubber like a real human ear, as shown Figure 4. In the experiments, the clip phone covers the KEMAR's pinna, and the error microphone is located inside the clip phone ear pad. The earbud earphone is placed on the outer ear, and the error microphone is attached on the earphone close to the ear canal. The in-ear earphone's plastic cap is inserted inside the KEMAR's ear canal. For comparison, we also place the commercial headsets-type A to cover the KEMAR's ear, type B to enclose the KEMAR's ear, and the plastic cap of the type C earphone is inserted inside the KEMAR's ear for measuring their performance. All the experiments are performed in a quiet room, and the primary noise is played at the same level for all experiments.

\subsection{Secondary Path Modeling Using Natural Sound}

We placed the light-weight ANC earphones on the KEMAR's ear to perform the secondary path modeling. We evaluated different natural sounds and decided to use a rain sound as the excitation signal for all earphones. Both white noise and natural sound used as the excitation signal were compared for estimating the secondary path $S(z)$. Only those music segments which have flat magnitude responses are applicable to identify the secondary path. In this paper, we have tested several natural sounds such as wave, water fall, the rain sound, etc. Among them, the rain sound has the similar spectrum with the white noise, shown in Figure 5a. So, we used the rain sound to identify the secondary path. Figure $5 b$ shows magnitude responses of the estimated secondary paths using the traditional white noise as the excitation signal (solid lines), and the results obtained using the rain natural sound (dotted line). Comparing the solid lines with the same color dotted lines, the results show the secondary path modeling using white noise and natural sound obtained similar magnitude responses, thus natural sound can be used for modeling. These results also show that the in-ear earphone has the best response in a low frequency range to achieve better noise reduction, which will be shown in the following subsection. 


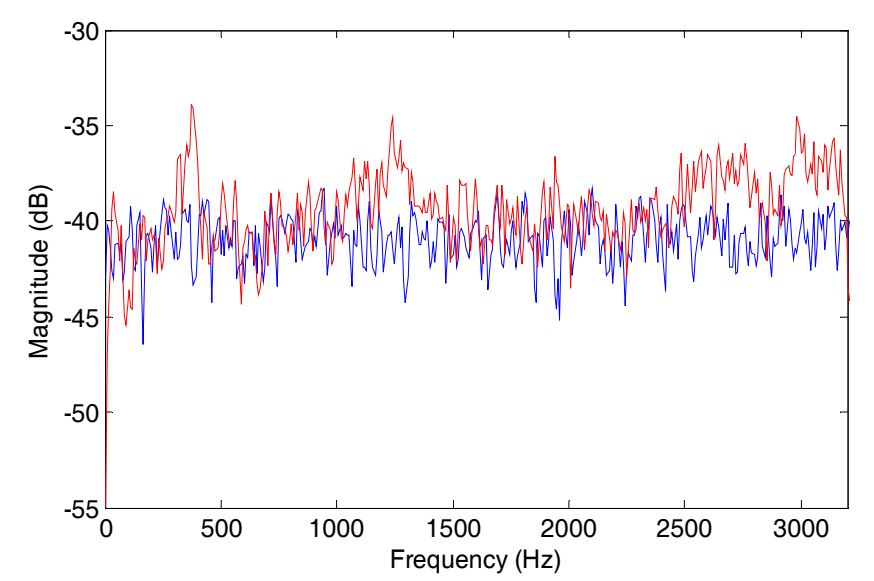

(a)

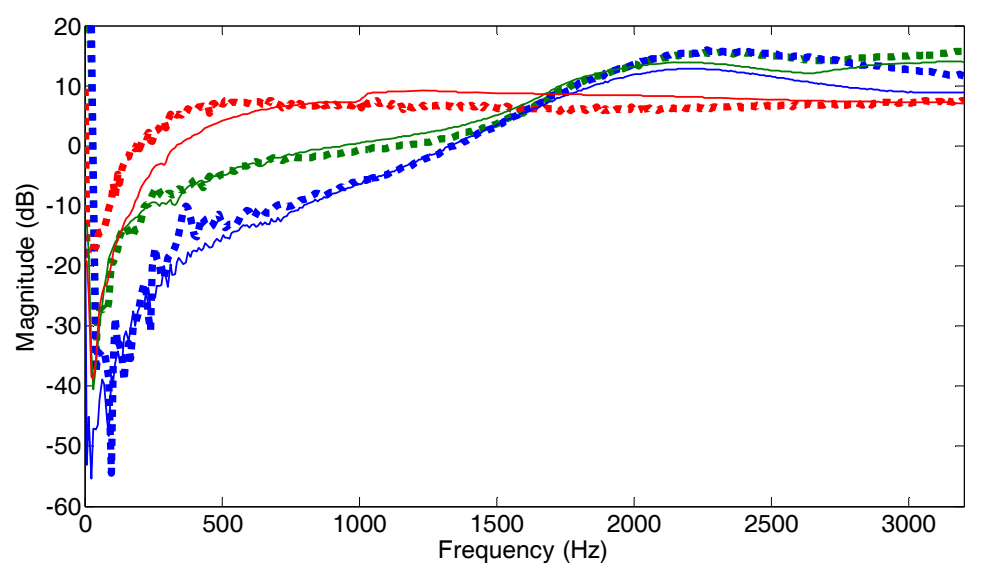

(b)

Figure 5. Magnitude responses, (a) the recorded rain sound (red line) and white noise (blue line); (b) results of $\hat{S}(z)$ using white noise (solid) and natural sound (dotted) for the in-ear (red), earbud (blue), and clip earphones (green).

\subsection{Real-Time Noise Reductions Results}

In the first set of experiments, we use stationary primary noise for evaluating noise reduction at different frequencies. As shown in Figure 4, the primary loudspeaker is used to generate single tone primary noise with frequency increasing from $100 \mathrm{~Hz}$ to $3000 \mathrm{~Hz}$ with the step of $100 \mathrm{~Hz}$. The light-weight ANC earphones are placed on the KEMAR's ear for experiment, and the identical experiments for the commercial ANC headphones and earphone (types A, B and C) were also repeated using the same noise level for comparison. The signal sensed by the KEMAR's microphone resembles the noise that will be perceived by the ANC earphones (or headphones) users. The power difference between the ANC OFF and ON is computed to obtain the noise reduction achieved by the ANC. The satisfactory performance can be achieved by using step size 0.0041 , filter length 100 for $\hat{S}(z)$ to model the secondary path, and the ANC filter $W(z)$ uses step size 0.0081 and filter length 450 .

Figure 6 summarizes the noise reduction achieved by six ANC earphones and headphones from $100 \mathrm{~Hz}$ to $3000 \mathrm{~Hz}$ with step of $100 \mathrm{~Hz}$. This clearly demonstrates that the developed light-weight ANC headphones achieved better noise reduction than the commercially available ANC headphones. Especially, the in-ear earphone obtained the best performance of noise reduction. The average noise reductions for the clip phones is $29 \mathrm{~dB}$, the earbud earphone is $35 \mathrm{~dB}$, and the in-ear earphone is $50 \mathrm{~dB}$. These three ANC earphones are effective over the wide frequency range from $100 \mathrm{~Hz}$ to $3000 \mathrm{~Hz}$. As a comparison, the commercial type A headphone failed to reduce noise for frequency above 
$1000 \mathrm{~Hz}$, the commercial type B headphone failed to reduce noise for frequency over $1500 \mathrm{~Hz}$, and the commercial type $\mathrm{C}$ earphone failed to reduce noise for frequency over $2200 \mathrm{~Hz}$.

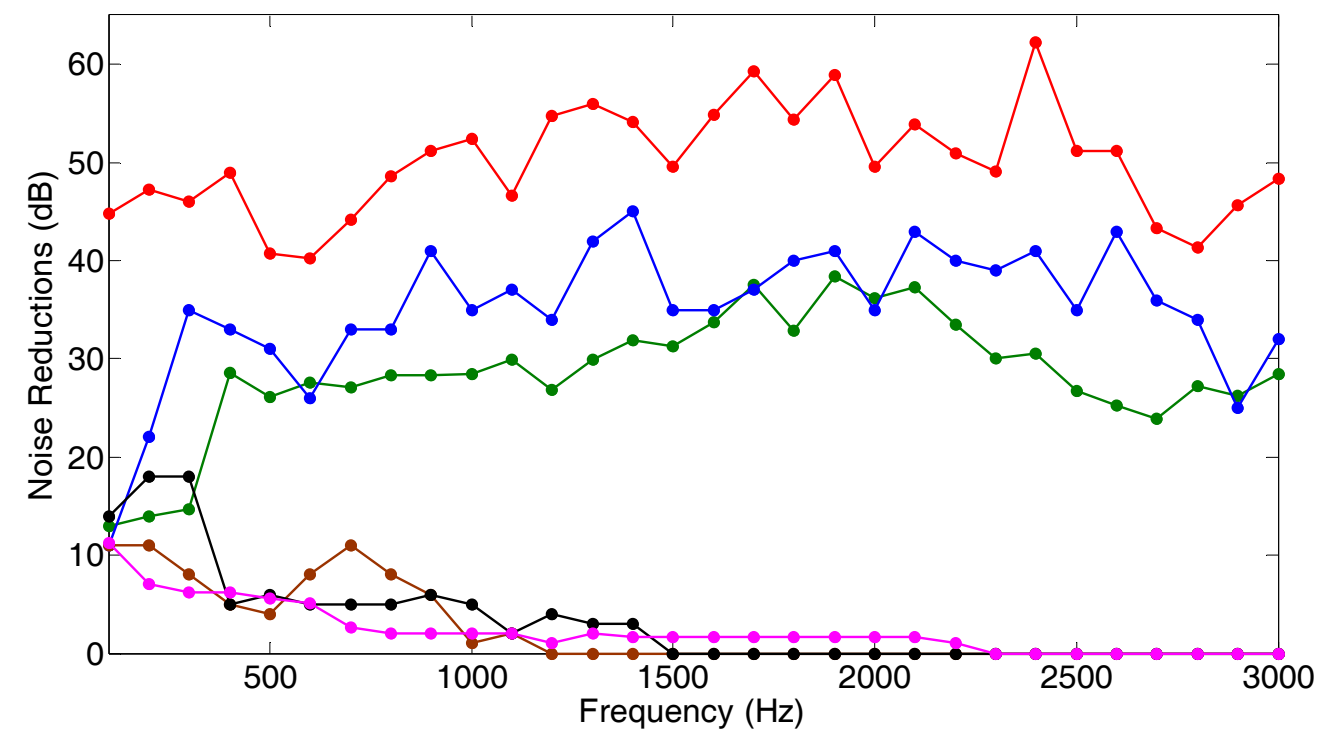

Figure 6. Reduction of tonal noise: clip (green), earbud (blue), and in-ear (red); the commercial type A (brown), type B (black), and type C (pink) earphones.

In the second set of experiments, the recorded MRI noise is used as the primary noise [16]. First, we measure the MRI noise at the KEMAR's ear without placing the earphone or headphone on it. After that, the developed light-weight ANC earphones and commercial ANC earphones and headphones are placed on the KEMAR to measure the MRI noise sensed by the KEMAR's microphone without turning on the ANC. This stage evaluates the passive noise reduction achieved by the earphones and headphones. Finally, we turn on the ANC and measure the residual noise for every ANC earphone and headphone.

Figure 7a shows the measured noise at the KEMAR's ear for the ANC clip earphones. It shows that the ANC clip earphone cannot passively attenuate the MRI noise, but there is $20 \mathrm{~dB}$ noise reduction from $1100 \mathrm{~Hz}$ to $3200 \mathrm{~Hz}$ after ANC is turned on. However, there is no noise reduction below $500 \mathrm{~Hz}$ due to the poor loudspeaker response at low frequency range as shown in Figure 5 of the clip earphone. Figure 7b shows the measured noise at the KEMAR's ear for the earbud ANC earphone. This shows the earbud earphone cannot passively attenuate the MRI noise neither, since the spectrum without placing the earphone is similar to the spectrum with earphone. After ANC is turned on, there is almost $20 \mathrm{~dB}$ noise reduction from $1000 \mathrm{~Hz}$ to $3200 \mathrm{~Hz}$. However, there is also no noise reduction below $500 \mathrm{~Hz}$ due to the poor loudspeaker response at low frequency range as shown in the blue lines of Figure 5 for the earbud ANC earphone. Figure 7c shows the measured noise spectra of the in-ear ANC earphone from these three steps. Comparing the black line and blue lines, we show that the in-ear earphone can attenuate high frequency MRI noise from $500 \mathrm{~Hz}$ to $2500 \mathrm{~Hz}$ by its plastic earpad without using ANC. In addition, comparing the blue line (ANC OFF) and the red line (ANC ON), there is about $15 \mathrm{~dB}$ noise reduction for frequency range up to $2400 \mathrm{~Hz}$, which is achieved by ANC. Since the in-ear ANC earphone's error microphone is inside the ear canal and its loudspeaker has good frequency response as shown in Figure 5 (red lines), it can reduce broadband noise efficiently. 


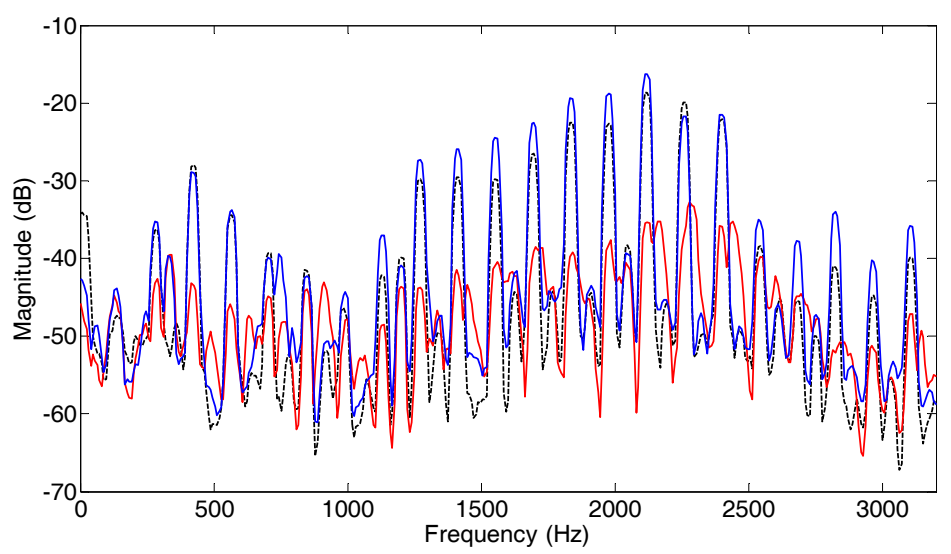

(a)

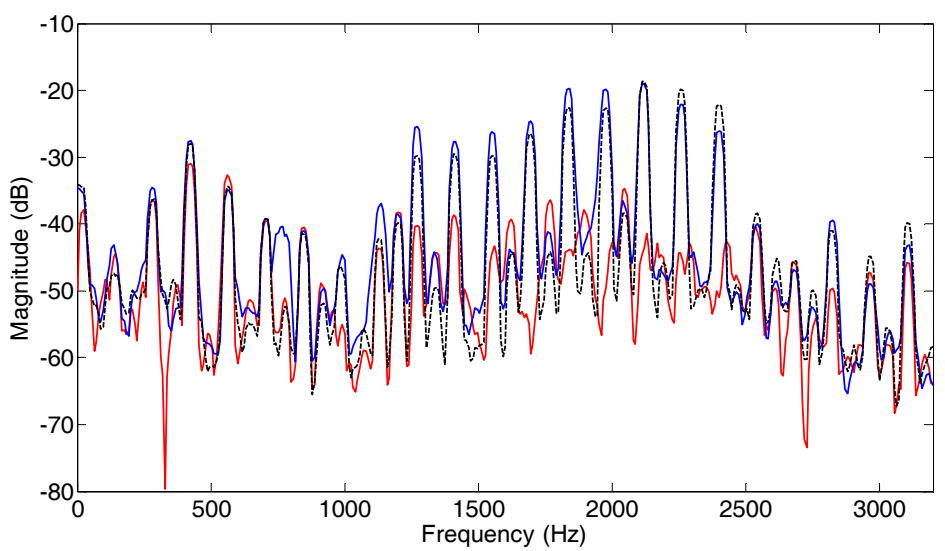

(b)

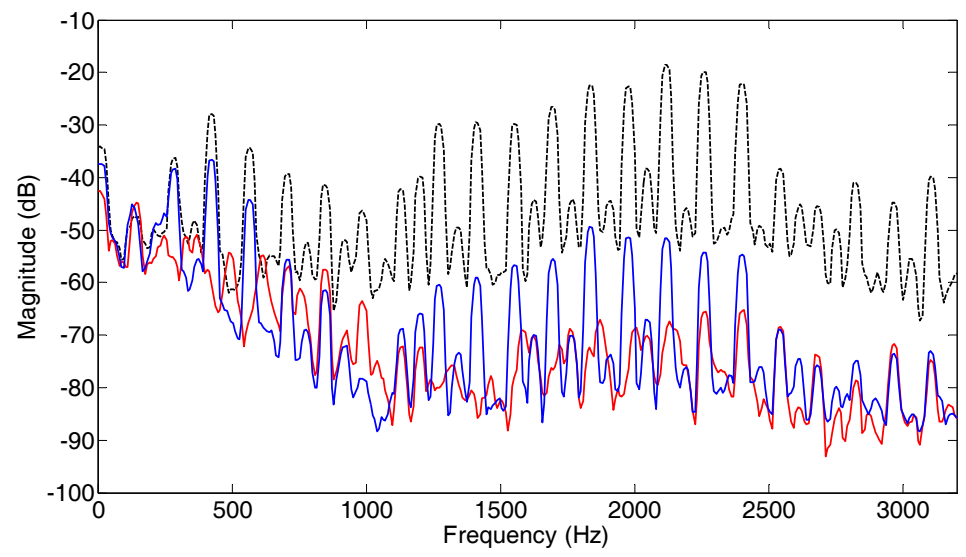

(c)

Figure 7. Spectra of noise measured at the KEMAR's ear without earphone on it (black), with earphone but without ANC (blue), and after ANC is turned on (red). (a) Clip earphone; (b) earbud earphone; (c) in-ear earphone.

Figure 8a shows the measured noise at the KEMAR's ear for the commercial type A headphone. It shows that the type A headphone cannot passively attenuate the MRI noise by its ear caps. After ANC is turned on, there is $10 \mathrm{~dB}$ noise reduction from $100 \mathrm{~Hz}$ to $500 \mathrm{~Hz}$. However, there is no noise reduction from $600 \mathrm{~Hz}$ to $3200 \mathrm{~Hz}$. The commercial type A headphone even amplifies $5 \mathrm{~dB}$ of MRI noise for high frequency from $1800 \mathrm{~Hz}$ to $2900 \mathrm{~Hz}$. Figure $8 \mathrm{~b}$ shows the measured noise at the KEMAR's ear for the commercial type B headphone. It shows that the commercial type B headphone can passively attenuate 
the MRI noise about $10 \mathrm{~dB}$ to $20 \mathrm{~dB}$ passively by its earmuffs from $300 \mathrm{~Hz}$ to $3200 \mathrm{~Hz}$. After ANC is turned on, there is $15 \mathrm{~dB}$ noise reduction from $100 \mathrm{~Hz}$ to $900 \mathrm{~Hz}$. However, the commercial type B headphone amplifies $2 \mathrm{~dB}$ to $5 \mathrm{~dB}$ of MRI noise from $900 \mathrm{~Hz}$ to $3200 \mathrm{~Hz}$. Figure $8 \mathrm{c}$ shows the measured noise at the KEMAR's ear for the commercial type $C$ in-ear earphone. It shows the type $C$ earphone can passively attenuate about $15 \mathrm{~dB}$ to $25 \mathrm{~dB}$ by its ear caps from $500 \mathrm{~Hz}$ to $3200 \mathrm{~Hz}$. After ANC is turned on, there is about $5 \mathrm{~dB}$ to $10 \mathrm{~dB}$ noise reduction from $100 \mathrm{~Hz}$ to $1000 \mathrm{~Hz}$, and $2 \mathrm{~dB}$ to $5 \mathrm{~dB}$ noise reduction from $1100 \mathrm{~Hz}$ to $3000 \mathrm{~Hz}$.

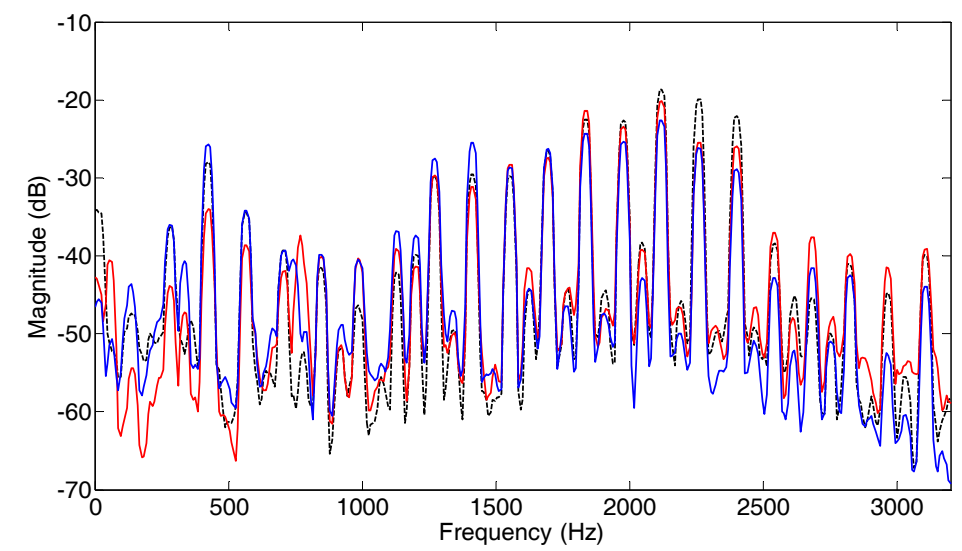

(a)

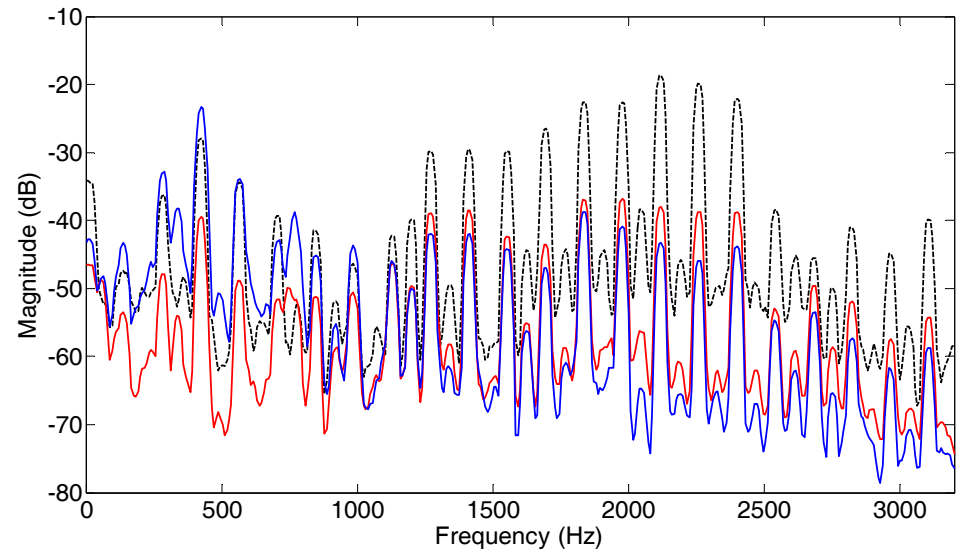

(b)

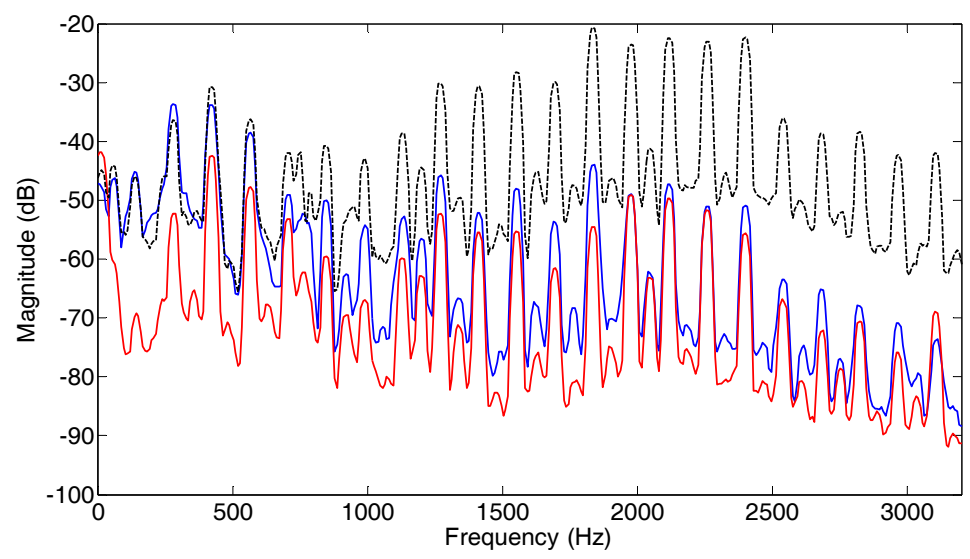

(c)

Figure 8. Spectra of noise measured at the KEMAR's ear without earphone on it (black), with earphone but without ANC (blue), and after ANC is turned on (red). (a) Type A; (b) type B; (c) type C. 
Comparing the similar ANC clip earphone (Figure 7a) with the type A headphone (Figure 8a), we found that neither can passively attenuate high frequency noise. However, when ANC is turned on, there is no noise reduction of the type A headphone beyond $500 \mathrm{~Hz}$, but there is $20 \mathrm{~dB}$ noise reduction of the developed ANC clip earphone. Therefore, the developed ANC clip earphone has better performance than the type A headphone. Besides, comparing the earbud ANC earphone (Figure $\mathrm{7b}$ ) with the commercial type B headphone (Figure $8 \mathrm{~b}$ ), both the earphones speakers are outside the ear canal. However, after ANC is turned on, the commercial type B headphone amplifies the noise from $900 \mathrm{~Hz}$ to $3200 \mathrm{~Hz}$, but the developed earbud earphone still performs well to cancel noise. Comparing the similar style of the developed in-ear ANC earphone (Figure 7c) with the commercial type $\mathrm{C}$ earphone (Figure $8 \mathrm{c}$ ), both are in-ear earphones and can passively attenuate high frequency MRI noise. However, the developed in-ear ANC earphone has better average noise reduction than the commercial type $\mathrm{C}$ earphone.

In summary, the ANC earphones developed by this paper are more efficient for noise reduction, and more portable and comfortable to wear than the ANC headphones, especially for outdoor activities. Besides, the developed light-weight ANC earphones only integrate error microphone and ANC filter, which is the same setup as commercial headsets. Thus, the price of the developed earphones in this paper should be acceptable as a consumer electronic device. The stability of the adaptive feedback ANC system can be assured by properly choosing the step size [17].

\section{Conclusions}

This paper developed three light-weight earphones with integration of DSP based ANC systems and compared their performance with three commercial ANC headphones and earphone. Experimental setup was carefully addressed for fair comparison. We also proposed using pleasant audio signals for modeling secondary path instead of annoying white noise, and evaluated its effectiveness. The light-weight ANC earphones developed by this paper are more comfortable to wear and achieved better noise reduction than the commercially available ANC headphones and earphone.

Author Contributions: Conceptualization and Writing-Review \& Editing, S.M.K.; Investigation, and WritingOriginal Draft Preparation, Y.-R.C.; Methodology, C.-Y.C.; Funding Acquisition, C.-W.L.

Conflicts of Interest: The authors declare no conflict of interest.

\section{References}

1. Fligor, B.J.; Levey, S.; Levey, T. Cultural and demographic factors influencing noise exposure estimates from use of portable listening devices in an urban environment. J. Speech Lang. Hear. Res. 2014, 57, 1535-1547. [CrossRef] [PubMed]

2. Hansen, C.H.; Snyder, S.D. Active Control of Noise and Vibration; E\&FN Spon: London, UK, 1997.

3. Kuo, S.M.; Morgan, D.R. Active Noise Control Systems: Algorithms and DSP Implementations; Wiley: New York, NY, USA, 1996.

4. Gan, W.S.; Kuo, S.M. An integrated audio and active noise control headset. IEEE Trans. Consum. Electron. 2002, 48, 242-247. [CrossRef]

5. Gan, W.S.; Mitra, S.; Kuo, S.M. Adaptive feedback active noise control headset: Implementation, evaluation and its extensions. IEEE Trans. Consum. Electron. 2005, 51, 975-982. [CrossRef]

6. Vu, H.S.; Chen, K.H. A low-power broad-bandwidth noise cancellation VLSI circuit design for in-ear headphones. IEEE Trans. Very Large Scale Integr. (VLSI) Syst. 2016, 24, 2013-2025. [CrossRef]

7. Guldenschuh, M.; de Callafon, R. Detection of secondary-path irregularities in active noise control headphones. IEEE/ACM Trans. Audio Speech Lang. Process. 2014, 22, 1148-1157. [CrossRef]

8. Chang, C.Y.; Li, S.T. Active noise control in headsets by using a low-cost microcontroller. IEEE Trans. Ind. Electron. 2011, 58, 1936-1942. [CrossRef]

9. Lim, D.H.; Kim, M.; Park, H.M. Active noise canceling for headphones using a hybrid structure with wind detection and flexible independent component analysis. IEICE Trans. Inf. Syst. 2015, E98D, 2043-2046. [CrossRef] 
10. Kuo, S.M.; Mitra, S.; Gan, W.S. Active noise control system for headphone applications. IEEE Trans. Control Syst. Technol. 2006, 14, 331-335. [CrossRef]

11. Song, Y.; Gong, Y.; Kuo, S.M. A robust hybrid feedback active noise cancellation headset. IEEE Trans. Speech Audio Process. 2005, 13, 607-617. [CrossRef]

12. HEADPHONES REVIEWS. Available online: https:/ / www.rtings.com/headphones (accessed on 1 July 2018).

13. Williams, W. Clamping pressure and circum-aural earmuffs. J. Noise Health 2007, 35, 45-50. [CrossRef]

14. Dan-Goor, E.; Samra, M. Benign paroxysmal positional vertigo after use of noise-canceling headphones. Am. J. Otolaryngol. 2012, 33, 364-366. [CrossRef] [PubMed]

15. Kuo, S.M.; Chuang, H.; Mallela, P. Integrated automotive signal processing and audio system. IEEE Trans. Consum. Electron. 1993, 39, 522-532. [CrossRef]

16. Kajikawa, Y. Feedback active noise control system and its application to MRI noise. In Proceedings of the 2011 8th International Conference on Information, Communications \& Signal Processing, Singapore, 13-16 December 2011; pp. 1-5.

17. Widrow, B.; Stearns, S.D. Adaptive Signal Processing; Prentice-Hall: Englewood Cliffs, NJ, USA, 1985.

(C) 2018 by the authors. Licensee MDPI, Basel, Switzerland. This article is an open access article distributed under the terms and conditions of the Creative Commons Attribution (CC BY) license (http://creativecommons.org/licenses/by/4.0/). 